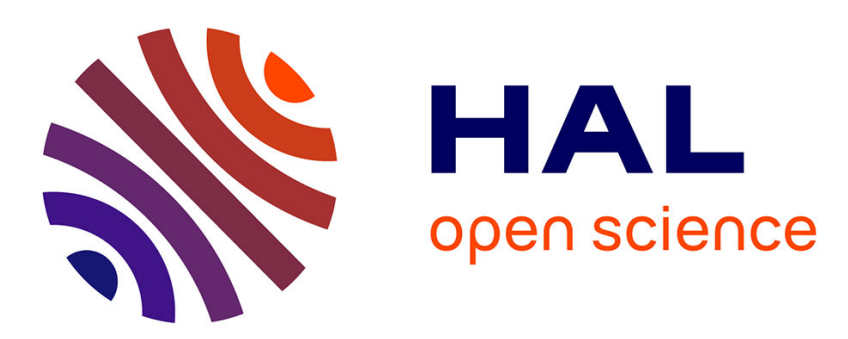

\title{
Time-homogenization of a first order system arising in the modelling of the dynamics of dislocation densities
}

\author{
Ariela Briani, Régis Monneau
}

\section{To cite this version:}

Ariela Briani, Régis Monneau. Time-homogenization of a first order system arising in the modelling of the dynamics of dislocation densities. Comptes rendus de l'Académie des sciences. Série I, Mathématique, 2009, 347, pp.231-236. 10.1016/j.crma.2009.01.006 . hal-00339921

\section{HAL Id: hal-00339921 \\ https://hal.science/hal-00339921}

Submitted on 19 Nov 2008

HAL is a multi-disciplinary open access archive for the deposit and dissemination of scientific research documents, whether they are published or not. The documents may come from teaching and research institutions in France or abroad, or from public or private research centers.
L'archive ouverte pluridisciplinaire HAL, est destinée au dépôt et à la diffusion de documents scientifiques de niveau recherche, publiés ou non, émanant des établissements d'enseignement et de recherche français ou étrangers, des laboratoires publics ou privés. 


\title{
Time-homogenization of a first order system arising in the modelling of the dynamics of dislocation densities
}

\author{
Ariela Briani ${ }^{\mathrm{a}}$, Régis Monneau ${ }^{\mathrm{b}}$ \\ a Dipartimento di Matematica, Largo Bruno Pontecorvo 5, 56127 Pisa, Italia. \\ ENSTA, 32 Boulevard Victor, 75739 Paris cedex 15, France. \\ ${ }^{\mathrm{b}}$ CERMICS, Paris Est-ENPC, 6 and 8 avenue Blaise Pascal, Cité Descartes, Champs sur Marne, 77455, Marne la \\ Vallée Cedex 2, France. \\ Received $* * * * *$; accepted after revision +++++ \\ Presented by
}

\begin{abstract}
In this Note we are interested in the dynamics of dislocation densities in a material submitted to a time periodic stress. The dislocation densities solve a set of two coupled first order equations of Burgers' type. Our main aim is to give a description of the long time behaviour of those densities. By an homogenization procedure in the framework of viscosity solutions, we obtain that at the limit, the dislocation densities fulfills a single diffusion equation.
\end{abstract}

To cite this article: A. Briani, R. Monneau, C. R. Acad. Sci. Paris, Ser. I 340 (2005).

\section{Résumé}

Homogénéisation en temps d'un système du premier ordre intervenant dans la modélisation de la dynamique de densités de dislocations. Dans cette Note, on s'intéresse à la dynamique de densités de dislocations dans un materiau soumis à un cisaillement périodique en temps. Ces densités sont solutions de deux équations couplées du premier ordre de type Burgers. Notre but est de décrire le comportement en temps long de ces densités. Nous développons une technique d'homogénéisation dans le cadre des solutions de viscosités, qui permet d'établir qu'à la limite les densités de dislocations sont solutions d'une seule équation de diffusion quasi-linéaire.

Pour citer cet article: A. Briani, R. Monneau, C. R. Acad. Sci. Paris, Ser. I 340 (2005).

Email addresses: briani@dm.unipi.it (Ariela Briani), monneau@cermics.enpc.fr (Régis Monneau). 


\section{Version française abrégée}

Les dislocations sont des défauts dans les cristaux qui bougent sous l'action d'une force extérieure. Elles sont considérées comme la principale explication à l'échelle microscopique de la plasticité des métaux. Dans [5], Groma et Balogh ont introduit un modéle 2D pour décrire la dynamique de densités de dislocations. Ce système peut être réduit, sous certaines hypothèses géométriques, à un système $1 \mathrm{D}$ de deux équations non locales de type transport (voir l'équation (1.1) dans [3]).

Ici on considère une version locale simplifiée et mise à l'échelle :

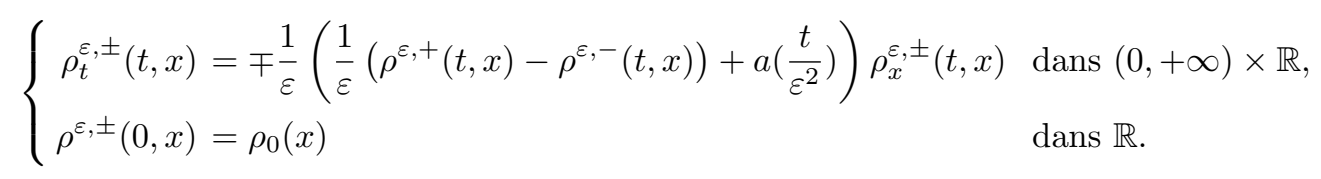

L'étude de la limite où le petit paramètre $\varepsilon>0$ tend vers zéro permet de décrire le comportement en temps long du système d'origine. De plus $\rho^{\varepsilon,+}-\rho^{\varepsilon,-}$ représente la déformation plastique du matériau, $\rho_{x}^{\varepsilon,+}$ et $\rho_{x}^{\varepsilon,-}$ sont les densités de dislocations associées réspectivement à un vecteur de Burgers de type positif ou negatif, $\left(\rho_{0}\right)_{x}$ est la densité positive ou nulle au temps initial et $a(\cdot)$ la force extérieure periodique.

On fait les hypothèses suivantes :

$(H a) \quad a: \mathbb{R} \rightarrow \mathbb{R}$ est une fonction $T$-périodique, non-nulle, telle que $\int_{0}^{T} a(\tau) d \tau=0$ et $a \in H_{l o c}^{2}(\mathbb{R})$.

$\left(H \rho_{0}\right) \quad \rho_{0}: \mathbb{R} \rightarrow \mathbb{R}$ peut être écrite, pour une constante $L_{0}$, comme $\rho_{0}(x)=P_{0}(x)+L_{0} x$ où $P_{0}$ est une fonction 1-périodique. On suppose aussi que $\left(\rho_{0}\right)_{x}(\cdot)=\left(P_{0}\right)_{x}(\cdot)+L_{0} \geq 0$ et $\left|\left(P_{0}\right)_{x x}(\cdot)\right| \leq C$.

Les fonctions $\rho^{\varepsilon}(t, x)=\left(\rho^{\varepsilon,+}(t, x), \rho^{\varepsilon,-}(t, x)\right)$ sont solutions du système (1) au sens de viscosité, ce système étant "quasi-monotone" (voir la Définition 2.2 dans [6] et [3]).

Notre résultat principal dans la limite où $\varepsilon$ tend vers zéro est le suivant.

Théorème 0.1 (Résultat d'homogénéisation) Sous les hypothèses $(\mathrm{Ha})$ et $\left(\mathrm{H} \rho_{0}\right)$, il existe une fonction continue $\bar{H}:[0,+\infty) \rightarrow \mathbb{R}$ telle que $\bar{H}(0)=0, \bar{H}(\theta)>0$ pour $\theta>0$, et $\lim _{\theta \rightarrow \infty} \bar{H}(\theta)=0$. Il existe une unique solution de viscosité $\rho^{\varepsilon}(t, x)=\left(\rho^{\varepsilon,+}(t, x), \rho^{\varepsilon,-}(t, x)\right)$ de $(1)$, Lipschitz en $x \in \mathbb{R}$, uniformément en $t>0$. De plus, lorsque $\varepsilon \rightarrow 0, \rho^{\varepsilon, \pm} \rightarrow \rho^{0}$ uniformément sur les compacts de $[0,+\infty) \times \mathbb{R}$, où $\rho^{0}(x, t)$ est l'unique solution de viscosité de

$$
\begin{cases}\rho_{t}^{0}(t, x)=\bar{H}\left(\rho_{x}^{0}(t, x)\right) \rho_{x x}^{0}(t, x) & \text { dans }(0,+\infty) \times \mathbb{R} \\ \rho^{0}(0, x)=\rho_{0}(x) & \text { dans } \mathbb{R} .\end{cases}
$$

Les deux densités $\rho_{x}^{\varepsilon, \pm}$ convergent donc vers la même densité $\rho_{x}^{0}$ qui vérifie formellement l'équation de diffusion obtenue en dérivant l'équation (2) par rapport à la variable d'espace.

La preuve de ce théoreme repose sur des arguments d'homogénéisation dans le cadre des solutions de viscosité. En particulier, on adapte la méthode de la fonction test perturbée introduite par Evans dans [4]. La preuve est détaillée en Section 3. L'idée principale est de décrire le comportement de $\rho^{\varepsilon, \pm}$ par un ansatz $\tilde{\rho}^{\varepsilon, \pm}$ que l'on suppose de la forme suivante : $\tilde{\rho}^{\varepsilon, \pm}(t, x)=\rho^{0}(t, x) \pm \varepsilon v^{1}\left(\frac{t}{\varepsilon^{2}}, \rho_{x}^{0}(t, x)\right)+$ $\varepsilon^{2} v^{2}\left(\frac{t}{\varepsilon^{2}}, \rho_{x}^{0}(t, x)\right) \rho_{x x}^{0}(t, x)$, où on doit définir les correcteurs $v^{1}$ et $v^{2}$ de telle sorte que $\tilde{\rho}^{\varepsilon, \pm} \rightarrow \rho^{0}$ lorsque $\varepsilon \rightarrow 0$. Pour la définition précise des correcteurs $v^{1}, v^{2}$ et de l'Hamiltonien effectif $\bar{H}$, nous renvoyons à la Proposition 2.1 en Section 2. 


\section{Introduction and main result.}

Dislocations are defects in crystals that are the main explanation at the microscopic scale of the plasticity of metals. Under an exterior shear stress the dislocations move in the material. In 1997 Groma and Balogh introduced a 2-D model to describe the dynamics of dislocation densities (see [5]). As shown by A. El Hajj and N. Forcadel in [3], and for a certain geometry invariant by translation in one direction, the 2-D model can be reduced to a 1-D system of coupled non-local transport equations (equation (1.1) in [3]). Dropping the non local term to simplify the analysis, this system reduces to a simple first order system. More precisely, fix $\varepsilon>0$, we consider the solution $\rho^{\varepsilon}(t, x)=\left(\rho^{\varepsilon,+}(t, x), \rho^{\varepsilon,-}(t, x)\right)$ of :

$$
\begin{cases}\rho_{t}^{\varepsilon, \pm}(t, x)=\mp \frac{1}{\varepsilon}\left(\frac{1}{\varepsilon}\left(\rho^{\varepsilon,+}(t, x)-\rho^{\varepsilon,-}(t, x)\right)+a\left(\frac{t}{\varepsilon^{2}}\right)\right) \rho_{x}^{\varepsilon, \pm}(t, x) & \text { in }(0,+\infty) \times \mathbb{R}, \\ \rho^{\varepsilon, \pm}(0, x)=\rho_{0}(x) & \text { in } \mathbb{R} .\end{cases}
$$

where $\rho^{\varepsilon,+}-\rho^{\varepsilon,-}$ represents the plastic deformation in the material, $\rho_{x}^{\varepsilon,+}$ and $\rho_{x}^{\varepsilon,-}$ the dislocation density respectively associated to a positive or negative Burgers vector (see [3]), $\left(\rho_{0}\right)_{x}$ the non negative density at the initial time and $a(\cdot)$ the exterior shear field. The parabolic rescaling $\tau=\frac{t}{\varepsilon^{2}}$ has been introduced to study the long time behaviour of the solution of the original system written in terms of $\tau$, with small exterior stress of order $O(\varepsilon)$.

On the data we will always assume:

$(H a) \quad a: \mathbb{R} \rightarrow \mathbb{R}$ is a non zero $T$-periodic function satisfying $\int_{0}^{T} a(\tau) d \tau=0$ and $a \in H_{\text {loc }}^{2}(\mathbb{R})$.

$\left(H \rho_{0}\right) \rho_{0}: \mathbb{R} \rightarrow \mathbb{R}$, can be written for some constant $L_{0}$ as $\rho_{0}(x)=P_{0}(x)+L_{0} x$ where $P_{0}$ is a 1 -periodic function. Moreover, we assume the following bounds: $\left(\rho_{0}\right)_{x}(\cdot)=\left(P_{0}\right)_{x}(\cdot)+L_{0} \geq 0$ and $\left|\left(P_{0}\right)_{x x}(\cdot)\right| \leq C$.

Assumption $\mathrm{Ha}$ ) on the periodicity of the stress $a$ can be interpreted in mechanics as a cyclic loading of the material. From $\left(H \rho_{0}\right)$, we see that $\left(\rho_{0}\right)_{x}$ is 1-periodic, which is a simplified assumption to study the solutions of (3) without taking into account any boundary effect in the material. This will particularly imply that the (non-negative) densities $\rho_{x}^{\varepsilon, \pm}(t, x)$ are also 1-periodic in $x$.

Solution of system (3) will always be understood in the viscosity sense for a "quasi-monotone" system (see Definition 2.2 in [6] and also [3]).

Our aim is to study the limit behaviour of the dislocation densities, i.e. to find the equation satisfied by the limit of the sequence $\left(\rho^{\varepsilon}\right)_{\varepsilon>0}$ as $\varepsilon \rightarrow 0$. The result is the following.

Theorem 1.1 (Homogenization result) Assume $(\mathrm{Ha})$ and $\left(H \rho_{0}\right)$.

There exists a continuous function $\bar{H}:[0,+\infty) \rightarrow \mathbb{R}$ such that $\bar{H}(0)=0, \bar{H}(\theta)>0$ for $\theta>0$, and $\lim _{\theta \rightarrow \infty} \bar{H}(\theta)=0$. There exists a unique $\rho^{\varepsilon}(t, x)=\left(\rho^{\varepsilon,+}(t, x), \rho^{\varepsilon,-}(t, x)\right)$ viscosity solution of (3) Lipschitz in $x \in \mathbb{R}$, uniformly in $t>0$. Moreover, $\rho^{\varepsilon, \pm} \rightarrow \rho^{0}$ uniformly on compact subsets of $[0,+\infty) \times \mathbb{R}$, as $\varepsilon \rightarrow 0$, where $\rho^{0}(t, x)$ is the unique viscosity solution of

$$
\begin{cases}\rho_{t}^{0}(t, x)=\bar{H}\left(\rho_{x}^{0}(t, x)\right) \rho_{x x}^{0}(t, x) & \text { in }(0,+\infty) \times \mathbb{R} \\ \rho^{0}(0, x)=\rho_{0}(x) & \text { in } \mathbb{R} .\end{cases}
$$

For the notion of viscosity solution for equation (4), we refer the reader to [2]. For a generalization of this result in the stochastic framework see [8].

Roughly speaking, this result tells us that the two densities $\rho_{x}^{\varepsilon, \pm}$ converge to the same $\rho_{x}^{0}$ which fulfills formally the diffusion equation obtained taking the space derivative of equation (4). Moreover, by the properties of the diffusion coefficient $\bar{H}$, we can say that when the density is very small or very high, the diffusion is very small. This can be thought as a first step in the understanding of the phenomenon of "Persistent Slip Bands" observed in experiments. Indeed, at a certain stage, dislocations are observed to 
form dislocation-rich and dislocation-poor regions with sometimes rather well defined spatial periodicity (see for instance [7]). This is also coherent with the phenomenum described in [1] where is explained that for fast moving dislocations "the effective current is diffusive on time scales longer than the period of the cyclic loading".

As an example of explicit $\bar{H}$, for $a(t)=A \cos (\omega t)$ with $A, \omega>0, T=\frac{2 \pi}{\omega}$, we get $\bar{H}(\theta)=\frac{\theta(A \omega)^{2}}{\omega^{2}+(2 \theta)^{2}}$.

The proof of Theorem 1.1 is done in the framework of viscosity solutions and is performed in Section 3. To this end, the notion of correctors is introduced in Section 2.

\section{The ansatz and definition of the correctors}

As usual in homogenization, we guess that the solutions $\rho^{\varepsilon, \pm}$ can be well approximated by an ansatz $\tilde{\rho}^{\varepsilon, \pm}$ that we assume to be of the following form:

$$
\tilde{\rho}^{\varepsilon, \pm}(t, x)=\rho^{0}(t, x) \pm \varepsilon v^{1}\left(\frac{t}{\varepsilon^{2}}, \rho_{x}^{0}(t, x)\right)+\varepsilon^{2} v^{2}\left(\frac{t}{\varepsilon^{2}}, \rho_{x}^{0}(t, x)\right) \rho_{x x}^{0}(t, x)
$$

where $v^{1}, v^{2}$ have to be determined in order to obtain $\tilde{\rho}^{\varepsilon, \pm} \rightarrow \rho^{0}$ as $\varepsilon \rightarrow 0$. To derive the definition of the correctors $v^{1}$ and $v^{2}$ in a formal way, we suppose "all the regularity we need", we define the new variables $\tau=\frac{t}{\varepsilon^{2}}, \theta=\rho_{x}^{0}$ and we plug $\tilde{\rho}^{\varepsilon, \pm}$ in system (3). We get

$\rho_{t}^{0}(t, x) \pm \frac{1}{\varepsilon} v_{\tau}^{1}(\tau, \theta)+v_{\tau}^{2}(\tau, \theta) \rho_{x x}^{0}(t, x) \approx \mp \frac{1}{\varepsilon}\left(2 v^{1}(\tau, \theta)+a(\tau)\right) \theta-\left(2 v^{1}(\tau, \theta)+a(\tau)\right) v_{\theta}^{1}(\tau, \theta) \rho_{x x}^{0}(t, x)+O(\varepsilon)$.

Identifying the terms of the same order, forgetting those of order $\varepsilon$ and setting $v^{3}:=v_{\theta}^{1}$, we obtain

$$
\begin{aligned}
v_{\tau}^{1}(\tau, \theta) & =-\left(2 v^{1}(\tau, \theta)+a(\tau)\right) \theta \\
\rho_{t}^{0}(t, x)+v_{\tau}^{2}(\tau, \theta) \rho_{x x}^{0}(t, x) & =-\left(2 v^{1}(\tau, \theta)+a(\tau)\right) v^{3}(\tau, \theta) \rho_{x x}^{0}(t, x) \\
v_{\tau}^{3}(\tau, \theta) & =-2 v^{3}(\tau, \theta) \theta-\left(2 v^{1}(\tau, \theta)+a(\tau)\right) .
\end{aligned}
$$

If we suppose that the functions $v^{i}(\cdot, \theta), i=1,2,3$, are $T$-periodic with null integral on the period, integrating $(7)$ and $(8)$ on $(0, T)$ we can formally derive the limit equation and the so-called effective Hamiltonian $\bar{H}$. We find:

$$
\rho_{t}^{0}(t, x)=\bar{H}\left(\rho_{x}^{0}(t, x)\right) \rho_{x x}^{0}(t, x) \quad \text { with } \quad \bar{H}(\theta)=\frac{2 \theta}{T} \int_{0}^{T}\left(v^{3}(s, \theta)\right)^{2} d s .
$$

The above calculation motivate the following definition.

Proposition 2.1 (Definition of the correctors) Assume $(H a)$. Fix $\theta \in[0,+\infty)$, the number $\bar{H}(\theta)$ is defined as the unique real number such that there are three T-periodic functions $v^{i}(\cdot, \theta), i=1,2,3$, with null integral on the period, solution of

$$
v_{\tau}^{1}=-\left(2 v^{1}+a\right) \theta, \quad v_{\tau}^{3}=-2 v^{3} \theta-\left(2 v^{1}+a\right), \quad \bar{H}(\theta)+v_{\tau}^{2}=-\left(2 v^{1}+a\right) v^{3} .
$$

Moreover, those solutions are unique and satisfy $v^{i} \in C^{1}(\mathbb{R} \times[0,+\infty)), i=1,2,3$. Furthermore, $\bar{H}(\theta)$ is a continuous function such that $\bar{H}(0)=0, \bar{H}(\theta)>0$ for $\theta>0$ and $\lim _{\theta \rightarrow \infty} \theta^{3} \bar{H}(\theta)=\frac{1}{8 T} \int_{0}^{T}\left(a_{\tau}(s)\right)^{2} d s$. In the proof of this Proposition we will need the following result which follows from Cauchy-Schwartz inequality. 
Lemma 1 Let $f \in L^{2}(0, T)$ be a T-periodic function such that $\int_{0}^{T} f(s) d s=0$. Fix $\bar{\varepsilon}>0$, there exists a unique T-periodic function $g$ with $\int_{0}^{T} g(s) d s=0$, solution of $g+\bar{\varepsilon} g_{\tau}=f$ and such that

$$
\|g\|_{2} \leq\|f\|_{2} \quad \text { and } \quad \text { if } f_{\tau} \in L^{2}(0, T) \text { then }\|g-f\|_{2} \leq \bar{\varepsilon}\left\|f_{\tau}\right\|_{2} \text {. }
$$

Proof of Proposition 2.1. Simply integrating explicitly the system we have that $\left(v^{1}, v^{2}, v^{3}\right)$ are $C^{1}(\mathbb{R} \times$ $[0,+\infty)), T$-periodic functions in $\tau$ with null integral on the period and we justify (9). Therefore, the only thing that remains to prove is the behaviour of $\bar{H}$ as $\theta$ goes to $\infty$. We set $\bar{v}^{3}:=v^{3} \theta^{2}$ and $\bar{a}:=\left(2 v^{1}+a\right) \theta$. The first two equations of (10) become $\bar{a}+\frac{1}{2 \theta} \bar{a}_{\tau}=\frac{a_{\tau}}{2}, \bar{v}^{3}+\frac{1}{2 \theta} \bar{v}_{\tau}^{3}=-\frac{\bar{a}}{2}$ and $\theta^{3} \bar{H}(\theta)=\frac{2}{T} \int_{0}^{T}\left(\bar{v}^{3}(s, \theta)\right)^{2} d s$. Applying Lemma 1 and assumption $(H a)$, we can see that $\left|\theta^{3} \bar{H}(\theta)-\frac{1}{8 T} \int_{0}^{T}\left(a_{\tau}\right)^{2}(s) d s\right| \rightarrow 0$ as $\theta \rightarrow \infty$. $\square$

\section{Proof of the homogenization result}

From now on, by "solution" will always mean "viscosity solution".

Proof of Theorem 1.1. For the existence and uniqueness of the solution $\rho^{\varepsilon}$ to (3) and $\rho^{0}$ to (4) (with $\bar{H}$ given in Proposition 2.1) we refer the reader respectively to Theorem 4.2 in [3] and to [2].

The proof of convergence will follow the classical method of building suitable perturbed test functions (see Evans [4]). We consider the following relaxed semi-limits of $\rho^{\varepsilon, \pm}$ :

$$
\bar{\rho}^{0}(t, x):=\limsup _{\varepsilon \rightarrow 0} \rho^{\varepsilon,+}(t, x) \vee \limsup _{\varepsilon \rightarrow 0} \rho^{\varepsilon,-}(t, x), \quad \underline{\rho}^{0}(t, x):=\liminf _{\varepsilon \rightarrow 0} \rho^{\varepsilon,+}(t, x) \wedge \liminf _{\varepsilon \rightarrow 0} \rho^{\varepsilon,-}(t, x)
$$

where $\limsup _{\varepsilon \rightarrow 0} \rho^{\varepsilon, \pm}(t, x):=\limsup _{(\tau, y) \rightarrow(t, x), \varepsilon \rightarrow 0} \rho^{\varepsilon, \pm}(\tau, y)$ and $\liminf _{\varepsilon \rightarrow 0} \rho^{\varepsilon, \pm}(t, x):=\liminf _{(\tau, y) \rightarrow(t, x), \varepsilon \rightarrow 0} \rho^{\varepsilon, \pm}(\tau, y)$.

We will proceed in four steps. In the first two steps we get some a priori estimates and in the last two steps we prove the convergence.

Step 1. Barriers on $\underline{\rho}^{0}$ and $\bar{\rho}^{0}$. We fix $\varepsilon>0$, set $\tau:=\frac{t}{\varepsilon^{2}}$ and define $u(\tau, x)$ as the solution of the o.d.e.

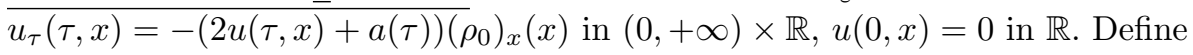

$$
\bar{b}^{\varepsilon, \pm}(t, x):=\rho_{0}(x) \pm \varepsilon u\left(\frac{t}{\varepsilon^{2}}, x\right)+\mu t \quad \text { and } \quad \underline{b}^{\varepsilon, \pm}(t, x):=\rho_{0}(x) \pm \varepsilon u\left(\frac{t}{\varepsilon^{2}}, x\right)-\mu t
$$

where $\mu:=\max \left\{\left|(2 u(\tau, x)+a(\tau)) u_{x}(\tau, x)\right|\right\}$ over $(\tau, x) \in[0,+\infty) \times \mathbb{R}$. Using the comparison principle, assumption $\left(H \rho_{0}\right)$ allows us to see that $\mu$ is finite. By calculation we get that $\underline{b}^{\varepsilon, \pm}$ and $\bar{b}^{\varepsilon, \pm}$ are respectively a sub and a supersolution of (3). Now, by the comparison principle (Theorem 4.2(i) in [3]) we have $\underline{b}^{\varepsilon, \pm}(t, x) \leq \rho^{\varepsilon, \pm}(t, x) \leq \bar{b}^{\varepsilon, \pm}(t, x)$, therefore, letting $\varepsilon \rightarrow 0$ we obtain the desired barriers: $\rho_{0}(x)-\mu t \leq \rho^{0}(t, x) \leq \bar{\rho}^{0}(t, x) \leq \rho_{0}(x)+\mu t$ in all $(0,+\infty) \times \mathbb{R}$.

Step 2. Monotonicity of $\underline{\rho}^{0}, \bar{\rho}^{0}$. Thanks to the positivity of $\left(\rho_{0}\right)_{x}$ in assumption $\left(H \rho_{0}\right)$, fix $h>0$, we $\overline{\text { have } \rho_{0}(x) \leq \rho_{0}(x+h) \text { for all } x} \in \mathbb{R}$. From the comparison principle we obtain $\rho^{\varepsilon, \pm}(t, x) \leq \rho^{\varepsilon, \pm}(t, x+h)$ for all $(t, x) \in(0,+\infty) \times \mathbb{R}$, thus, letting $\varepsilon \rightarrow 0$ we get $\left(\rho^{0}\right)_{x} \geq 0$ and $\left(\bar{\rho}^{0}\right)_{x} \geq 0$.

Step 3. $\bar{\rho}^{0}$ is a subsolution of (4). We argue by contradiction: let $\phi^{0} \in C^{\infty}$ be a test function such that $\overline{\bar{\rho}^{0}-\phi^{0} \text { has a strict local maximum }}$ at $\left(t_{0}, x_{0}\right)$, more precisely there exist $r>0$ and $\lambda>0$, such that

$$
\bar{\rho}^{0}\left(t_{0}, x_{0}\right)-\phi^{0}\left(t_{0}, x_{0}\right)=0, \quad \text { and } \quad \bar{\rho}^{0}(t, x) \leq \phi^{0}(t, x)-2 \lambda \text { on } \partial B_{r}\left(t_{0}, x_{0}\right) .
$$

We suppose that there is a $\eta>0$ such that

$$
\phi_{t}^{0}\left(t_{0}, x_{0}\right)=\bar{H}\left(\phi_{x}^{0}\left(t_{0}, x_{0}\right)\right) \phi_{x x}^{0}\left(t_{0}, x_{0}\right)+\eta \text {. }
$$


Fix $\varepsilon>0$, similarly to the ansatz (5) we define the perturbed test functions:

$\phi^{\varepsilon, \pm}(t, x):=\phi^{0}(t, x) \pm \varepsilon v^{1}\left(\frac{t}{\varepsilon^{2}}, \phi_{x}^{0}(t, x)\right)+\varepsilon^{2} v^{2}\left(\frac{t}{\varepsilon^{2}}, \phi_{x}^{0}(t, x)\right) \phi_{x x}^{0}(t, x)$, where $v^{1}, v^{2}$, are the solutions of (10) in Proposition 2.1 with the choice $\theta=\phi_{x}^{0}(t, x) \geq 0$ by Step 2 .

Step 3.1. $\phi^{\varepsilon, \pm}$ is a supersolution of (3) in a ball $B_{r}\left(t_{0}, x_{0}\right)$ with $r$ independent of $\varepsilon$. A direct computation shows that $\phi^{\varepsilon, \pm}$ is a supersolution of (3) if and only if

$$
\phi_{t}^{0}(t, x)+O(\varepsilon) \geq \pm \frac{1}{\varepsilon}\left\{\left(2 v^{1}(\tau, \theta)+a(\tau)\right) \theta-v_{\tau}^{1}(\tau, \theta)\right\}-\phi_{x x}^{0}(t, x)\left\{v_{\tau}^{2}(\tau, \theta)+\left(2 v^{1}(\tau, \theta)+a(\tau)\right) v_{\theta}^{1}(\tau, \theta)\right\},
$$

where $\tau:=\frac{t}{\varepsilon^{2}}$ and $\theta=\phi_{x}^{0}(t, x)$.

By definition of the correctors this is equivalent to $\phi_{t}^{0}(t, x)+O(\varepsilon) \geq \phi_{x x}^{0}(t, x) \bar{H}\left(\phi_{x}^{0}(t, x)\right)$ which is true in a neighborhood of $\left(t_{0}, x_{0}\right)$ because of (12).

Step 3.2. Contradiction. Because of (11) we deduce that $\rho^{\varepsilon, \pm}(t, x) \leq \phi^{\varepsilon, \pm}(t, x)-\lambda$ on $\partial B_{r}\left(t_{0}, x_{0}\right)$ for $\varepsilon$ small enough. From the comparison principle on balls (see Theorem 4.7 in [6]) we deduce that $\rho^{\varepsilon, \pm}(t, x) \leq$ $\phi^{\varepsilon, \pm}(t, x)-\lambda$ in all $B_{r}\left(t_{0}, x_{0}\right)$. Letting $\varepsilon \rightarrow 0$ we get $0=\bar{\rho}^{0}\left(x_{0}, t_{0}\right)-\phi^{0}\left(x_{0}, t_{0}\right) \leq-\lambda<0$ which yields to a contradiction.

Step 4. Conclusion. Similarly we prove that $\rho^{0}$ is a supersolution of (4). Then the conclusion that $\bar{\rho}^{0}=$ $\rho^{0}:=\rho^{0}$ is the solution of (4) is classical (using the barriers in Step 1 and the comparison principle for equation (4)).

\section{Acknowledgements}

The authors would like to thank Pierre Cardaliaguet for many stimulating discussions on this problem and a common reflection on a possible generalization of these results.

\section{References}

[1] E. C. Aifantis, D.Walgraef, Dislocation Patterning as the Result of Dynamical Instabilities, J.Appl.Phys. 58 (1985) 688-91.

[2] M. G. Crandall, H. Ishii, P.L. Lions, User's guide to viscosity solutions of second order partial differential equations, Bull. Amer. Math. Soc.(N.S.) 27 (1) (1992) 1-67.

[3] A. El Hajj, N. Forcadel, A convergent scheme for a non-local coupled system modelling dislocations densities dynamics, Math. Comp. 77 (262) (2008) 789-812.

[4] L. C. Evans, The perturbed test function-method for viscosity solutions of nonlinear partial differential equations, Proc. Roy. Soc. Edimburgh Sect. A 111 (1989) 359-375.

[5] I. Groma, P. Balogh, Investigation of dislocation pattern formation in a two-dimensional self-consistent field approximation, Acta mater. 47 (13) (1999) 3647-3654.

[6] H.Ishii, S. Koike, Viscosity solutions for monotone systems of second order elliptic pdes, Commun. in partial differential equations $16(6,7)(1991)$ 1095-1128.

[7] L. P. Kubin, C. Fressengeas, G. Ananthakrishna, Collective behaviour of dislocations in plasticity, in: F.R.N. Nabarro, M.S. Duesbery (Eds.), Dislocation of Solids 11, Elsevier Science B.V. 2002, 101-192.

[8] R. Monneau, P.E. Souganidis, Infinite Laplacian diffusion equations by stochastic time homogenization of a coupled system of first order equations, in preparation. 